\title{
Relation of Plasma Renin to Blood Pressure in a Patient with Coarctation
}

\author{
P. T. PICKENS* \\ From the Cleveland Clinic, Cleveland, Ohio, U.S.A.
}

Among several factors determining secretion of renin is mean arterial pressure (Skinner, McCubbin, and Page, 1964). Plasma renin is, in some instances, increased in renal artery stenosis (Helmer, 1964; Brown et al., 1964; Veyrat et al., 1964): and it is probable that this increase is due to lowered arterial pressure beyond the obstruction. In coarctation of the aorta there is a similar discrepancy between the pressure at which arterial blood is supplied to the kidney and that at which it is supplied to the upper part of the body. It is rather difficult to measure the pressure beyond a renal artery stenosis, but fairly simple to measure that distal to a coarctation. It seemed likely that in coarctation (and in renal artery stenosis) the control of renin secretion would be normal but appropriate to arterial pressure in the kidney rather than to brachial arterial pressure. Observations of plasma renin, brachial, and femoral arterial pressure were made in a patient with coarctation before and after surgical repair: measurements were also made before and after intravenous infusion of sodium nitroprusside (Page et al., 1955), in order that plasma renin levels might be compared at various arterial pressures.

\section{Case Report}

A 17-year-old-boy was found at routine medical examination to be hypertensive: he was free from symptoms, his exercise tolerance was normal, there was no past history of renal disease, and no family history of hypertension. He was referred to the Cleveland Clinic for further investigation. On examination the blood pressure was $180 / 90 \mathrm{~mm}$. Hg: the apex beat was within the mid-clavicular line. The heart sounds were normal: there was a harsh systolic murmur at the left sternal edge, heard also below the left clavicle and between the shoulder blades. The femoral pulses were weak and delayed. No other abnormality was found on clinical

\footnotetext{
^ Present address: Cardiff Royal Infirmary, Cardiff, Glam.
}

examination. Investigation showed renal function to be normal: the electrocardiogram showed left ventricular hypertrophy, and the chest radiograph showed slight left ventricular enlargement. Angiography demonstrated a coarctation of the aorta immediately distal to the origin of the left subclavian artery. The coarctation was resected and an end-to-end anastomosis performed.

Samples of plasma from peripheral venous blood were obtained for estimation of renin activity (Pickens $e t$ al., 1965) before and after operation. Plasma samples were also obtained after a fall in arterial pressure induced by intravenous infusion of sodium nitroprusside. The blood pressure was measured by sphygmomanometer in the arm and in the leg (using a wide cuff) at the time each blood sample was taken. It was established by direct reading from an intra-arterial needle that the mean pressure obtained in this way was similar to that obtained by calculation (diastolic pressure plus one-third pulse pressure) from measurements made with the sphygmomanometer.

In Fig. 1a plasma renin activity (nanograms of angiotensin generated per $10 \mathrm{ml}$. plasma after 4 hours incubation at $\mathrm{pH} 5.5,37^{\circ} \mathrm{C}$.) is related to mean arterial pressure. In Fig. $1 \mathrm{~b}$ the same readings of plasma renin activity are plotted against mean femoral arterial pressure. In the leg there is clearly an inverse relation between mean arterial pressure and plasma renin activity. By contrast, in the arm, before operation, the pressure would have led one to expect a plasma renin level much lower than that recorded. Had only brachial arterial pressure been measured it would have appeared, therefore, that the plasma renin level was inappropriately high.

\section{Comment}

It is recognized that the method used measures renin activity as it may be affected by other factors in the plasma (Pickens et al., 1965); and that mean arterial pressure is not the sole determinant of renin secretion. Nevertheless, it is suggested that the present observations are consonant with the view that the renin-secreting apparatus was functioning normally in this instance but was maintaining a 

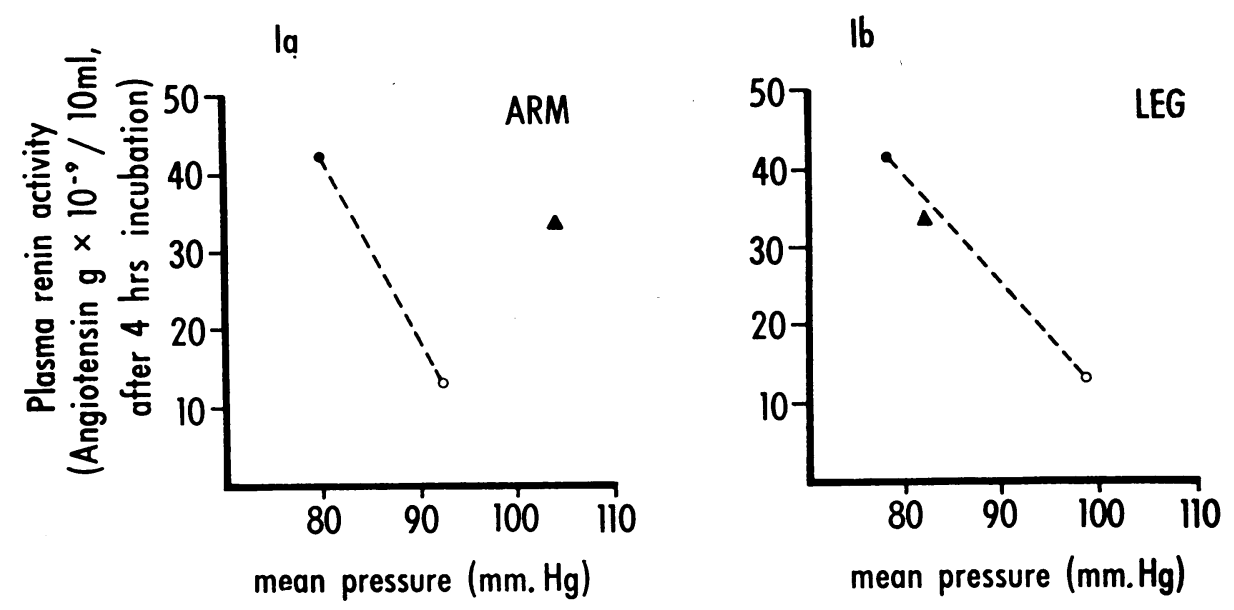

FIG. Relation of plasma renin activity to mean pressure in the arm and in the leg. $\Delta$, before operation; $O$, after operation; $\bullet$, after operation, following nitroprusside infusion.

plasma renin level appropriate to the pressure distal to the coarctation and hence inappropriate to brachial arterial pressure. It is noted that plasma renin activity fell after repair of the coarctation: it is not known to what extent the higher plasma renin may have been a cause of the higher blood pressure.

\section{Summary}

Plasma renin activity was measured in a patient with coarctation of the aorta, before and after surgical repair. Before operation the plasma renin level appeared inappropriately high if related to the arterial pressure in the arm. It is suggested that this was due to the discrepancy between arterial pressure in the arm and that in the renal artery: a similar discrepancy may account for the high plasma renin activity found in some patients with renal artery stenosis.

I am grateful for permission to publish these observations made on a patient under the care of Dr. I. H. Page,
Dr. H. P. Dustan, and Dr. Larry Groves, who performed the operation. I also gratefully acknowledge the assistance of Dr. Tom Meaney and other members of the Radiological Department of the Cleveland Clinic.

\section{References}

Brown, J. J., Davies, D. L., Lever, A. F., and Robertson, J. I. S. (1964). Variation in plasma renin concentration in several physiological and pathological states. Canad. med. Ass. F., 90, 201.

Helmer, O. M. (1964). Renin activity in blood from patients with hypertension. Canad. med. Ass. F., 90, 221.

Page, I. H., Corcoran, A. C., Dustan, H. P., and Koppanyi, T. (1955). Cardiovascular actions of sodium nitroprusside in animals and hypertensive patients. Circulation, 11, 188.

Pickens, P. T., Bumpus, F. M., Lloyd, A. M., Smeby, R. R., and Page, I. H. (1965). Measurement of renin activity in human plasma. Circulat. Res., 17, 438.

Skinner, S. L., McCubbin, J. W., and Page, I. H. (1964). Renal baroreceptor control of acute renin release in normotensive, nephrogenic and neurogenic hypertensive dogs. Circulat. Res., 15, 522.

Veyrat, R., de Champlain, J., Boucher, R., and Genest, J. (1964). Measurement of human arterial renin activity in some physiological and pathological states. Canad. med. Ass. F., 90, 215. 